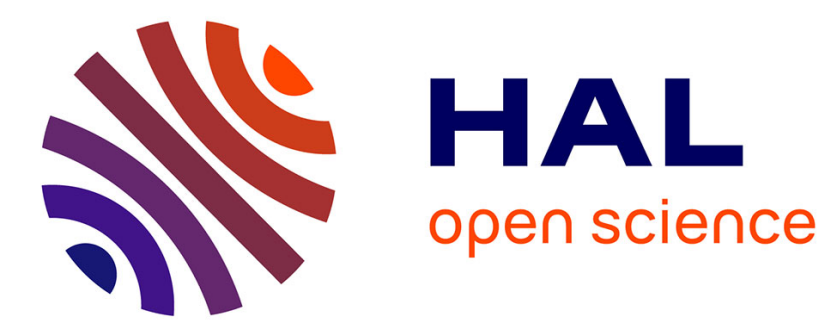

\title{
Propagation d'ondes le long de réseaux de tuyauteries
}

C. Biben, S. Pauzin, F. Simon

\section{To cite this version:}

C. Biben, S. Pauzin, F. Simon. Propagation d'ondes le long de réseaux de tuyauteries. Journal de Physique IV Proceedings, 1994, 04 (C5), pp.C5-69-C5-72. 10.1051/jp4:1994507 ～jpa-00252769

\section{HAL Id: jpa-00252769 https://hal.science/jpa-00252769}

Submitted on 1 Jan 1994

HAL is a multi-disciplinary open access archive for the deposit and dissemination of scientific research documents, whether they are published or not. The documents may come from teaching and research institutions in France or abroad, or from public or private research centers.
L'archive ouverte pluridisciplinaire HAL, est destinée au dépôt et à la diffusion de documents scientifiques de niveau recherche, publiés ou non, émanant des établissements d'enseignement et de recherche français ou étrangers, des laboratoires publics ou privés. 


\title{
Propagation d'ondes le long de réseaux de tuyauteries
}

\author{
C. BIBEN, S. PAUZIN et F. SIMON
}

CERT/ONERA/DERMES, 2 Avenue E. Belin, BP. 4025, 31055 Toulouse cedex, France

\begin{abstract}
In order to understand the shocks mechanisms propagation, the CERT / ONERA / DERMES is interested in structural waves propagation in piping system network. The free vibration of a cylindrical thin-walled shell can be described by the Donnell-Mushtari theory, added to Flügge formulation. The resolution of this equations system leads to a 6 order equation in $\omega$ (motion frequency). In this way, we obtain the axial, radial and circumferential propagation modes eigenfrequencies. However, due to the hypothesis of long pipe assumption, only the radial mode is taken into account. The theoretical approach is completed by an experimental study. Thus, the waves propagation along straight pipes and in complex system (in 3-D) including some representative discontinuities of system network (single $45^{\circ}$ bend, single $90^{\circ}$ bend, T-Joint, curved-pipe...) is analyzed. The aim is to quantify the amplitude attenuation of a radial propagation mode of the shell through these kind of discontinuities. The study is carried out in order to realize a numerical simulation software of vibrational waves propagation along piping system network. This study is financed by a natural gas transmission company : Gaz du Sud-Ouest.
\end{abstract}

\section{1 - INTRODUCTION :}

Dans le cadre de l'étude financée par le Gaz du Sud-Ouest, nous nous intéressons à la propagation d'onde de structures dans les systèmes de tuyauteries, afin de comprendre les mécanismes de propagation de chocs dans de tels systèmes.

Dans le cas de conduites cylindriques satisfaisant à l'hypothèse des coques minces, il se propage dans la structure, suite à une excitation de nature impulsionnelle, différents types d'ondes. On s'intéresse plus particulièrement aux ondes de flexion. La théorie utilisée pour décrire ces phénomènes est celle de Donnell-Mushtari.

En parallèle à cette étude théorique, des travaux expérimentaux sont menés afin d'analyser la propagation de telles ondes dans des conduites droites ou dans des systèmes complexes possédant diverses discontinuités pouvant être rencontrées sur un réseau.

\section{2 - ETUDE DES COQUES CYLINDRIQUES :}

\section{1 - Propagation des différentes ondes :}

Si l'on considère une coque mince cylindrique soumise à une excitation mécanique extérieure orientée suivant le rayon du cylindre, le déplacement radial est alors prédominant et la déformation consiste principalement en une flexion [1]. D'autre part, si la forme et les dimensions de la coque sont grandes devant la longueur d'onde, il se propage un type d'onde appelé "ondes quasi-longitudinales". La vitesse de telles ondes est alors constante et identique à celle d'ondes qui se propageraient dans une plaque plane, 
d'épaisseur égale à celle de la coque. Cette vitesse ne dépend que des caractéristiques physiques de la structure (module de Young, coefficient de Poisson, densité), contrairement aux ondes de flexion dont la célérité dépend de la fréquence (et donc du mode considéré), des conditions aux limites, du type d'excitation...[2]. Lors d'une excitation mécanique, ces deux types d'ondes coexistent et sont responsables des mouvements de déformation de la paroi de la coque.

\section{2 - Equations du mouvement :}

Pour décrire les mouvements de la paroi d'une coque mince cylindrique suivant les 3 directions axiale (x), azimutale $(\theta)$ et radiale $(r)$ on utilise les équations de Donnell-Mushtari qui sont basées sur les hypothèses établies par Love dans sa théorie sur les petits déplacements des coques minces. Ces équations sont complétées par la formulation de Flügge.

Le système d'équations à résoudre (écrit sous forme matricielle) est alors le suivant [3]:

$$
\left[\begin{array}{ccc}
\frac{\partial^{2}}{\partial s^{2}}+\left(1+\beta^{2}\right) \cdot\left(\frac{1-v}{2}\right) \cdot \frac{\partial^{2}}{\partial \theta^{2}}-\rho\left(\frac{1-v^{2}}{E}\right) \cdot R^{2} \cdot \frac{\partial^{2}}{\partial t^{2}} & \left(\frac{1+v}{2}\right) \cdot \frac{\partial^{2}}{\partial \delta \partial \theta} & v \cdot \frac{\partial}{\partial s}-\beta^{2} \cdot \frac{\partial^{3}}{\partial s^{3}}+\beta^{2} \cdot\left(\frac{1-v}{2}\right) \cdot \frac{\partial^{3}}{\partial s \partial \theta^{2}} \\
\left(\frac{1+v}{2}\right) \cdot \frac{\partial^{2}}{\partial s \partial \theta} & \left(1+\beta^{2}\right) \cdot\left(\frac{1-v}{2}\right) \cdot \frac{\partial^{2}}{\partial z^{2}}+\frac{\partial^{2}}{\partial \theta^{2}}-\rho\left(\frac{1-v^{2}}{E}\right) \cdot R^{2} \cdot \frac{\partial^{2}}{\partial t^{2}} & \frac{\partial}{\partial \theta}-\beta^{2} \cdot\left(\frac{3-v}{2}\right) \cdot \frac{\partial^{3}}{\partial s^{2} \partial \theta} \\
v \cdot \frac{\partial}{\partial s}-\beta^{2} \cdot \frac{\partial^{3}}{\partial s^{3}}+\beta^{2} \cdot\left(\frac{1-v}{2}\right) \cdot \frac{\partial^{3}}{\partial \delta \partial \theta^{2}} & \frac{\partial}{\partial \theta}-\beta^{2} \cdot\left(\frac{3-v}{2}\right) \cdot \frac{\partial^{3}}{\partial s^{2} \partial \theta} & 1+\beta^{2}+\beta^{2} \cdot \nabla^{4}+2 \cdot \beta^{2} \cdot \frac{\partial^{2}}{\partial \theta^{2}}+\rho\left(\frac{1-v^{2}}{E}\right) \cdot R^{2} \cdot \frac{\partial^{2}}{\partial t^{2}}
\end{array}\right] \cdot\left[\begin{array}{l}
u \\
v \\
w
\end{array}\right]=\{0\}
$$

avec : $(\mathrm{u}, \mathrm{v}, \mathrm{w})$ : composantes du déplacement suivant les directions $(\mathrm{x}, \boldsymbol{\theta}, \mathrm{r})$

$\mathrm{S}=\mathrm{x} / \mathrm{R}$ : variable adimensionnée

$\mathrm{R}$ : rayon moyen du cylindre

$E, v, \rho:$ module de Young, coefficient de Poisson, densité de la structure

$\beta^{2}$ : paramètre d'épaisseur du tube (adimensionné)

$\nabla^{4}=\nabla^{2} \cdot \nabla^{2} \quad$ et $\nabla^{2} \equiv \frac{\partial^{2}}{\partial s^{2}}+\frac{\partial^{2}}{\partial \theta^{2}}$

L'introduction dans cette équation matricielle d'un champ de déplacement de la forme :

$$
\left\{\begin{array}{l}
\mathrm{u}=\mathrm{U} \cdot \cos (\mathrm{n} \theta) \cdot \mathrm{e}^{-\mathrm{ikx}} \cdot \mathrm{e}^{\mathrm{i} \omega t} \\
\mathrm{v}=\mathrm{V} \cdot \sin (\mathrm{n} \theta) \cdot \mathrm{e}^{-\mathrm{ikx}} \cdot \mathrm{e}^{\mathrm{i} \omega t} \\
\mathrm{w}=\mathrm{W} \cdot \cos (\mathrm{n} \theta) \cdot \mathrm{e}^{-\mathrm{ikx}} \cdot \mathrm{e}^{\mathrm{i} \omega t}
\end{array}\right.
$$

avec : $(\mathrm{U}, \mathrm{V}, \mathrm{W})$ : constantes

$\mathrm{n}$ : ordre du mode de propagation radial

$\mathbf{k}$ : nombre d'onde axial

nous conduit à l'équation de dispersion. Celle-ci peut s'écrire sous deux formes distinctes, soit une équation du $6^{\text {ième }}$ ordre en $\omega$ (avec $\mathrm{k}$ et $\mathbf{n}$ fixés), soit une équation du $8^{\text {ième }}$ ordre en $\mathrm{k}$ ( $\omega$ et $\mathbf{n}$ fixés).

\section{3 - Relation de dispersion :}

Si l'on considère l'équation du 6ième ordre en $\omega$, la résolution de celle-ci nous permet de connaître pour une coque donnée, les fréquences propres des modes de propagation transverse. Un processus informatique de calcul des racines d'un polynôme a été utilisé pour trouver ces fréquences. Ce programme prend en compte les modes couplés longitudinaux et transverses, et ce dans le cas d'une coque cylindrique de longueur variable placée en conditions libre-libre ou appuyée-appuyée. On introduit dans le système d'équations aux dérivées partielles ci-dessus, les équations de conditions aux limites (quatre à chaque extrémité). Ce programme à été validé par comparaison à des résultats obtenus lors d'essais par $C$. $Y$. Glandier [4]. Les formes propres du cylindre sont supposées identiques à celles d'une poutre. En ce qui concerne le cas appuyé-appuyé, il vérifie totalement les conditions aux limites imposées au cylindre ainsi que les équations du mouvement, ce qui n'est pas le cas des conditions libre-libre.

Nous donnons dans le tableau ci-après (Tableau 1) les valeurs des fréquences propres obtenues pour le mode transverse $n=2$ ("Ovalling mode") dans le cas d'une conduite cylindrique (en acier) dont les 
caractéristiques sont définies ci-dessous. Ce mode à été identifié comme un des plus énergétique lors de premiers essais.

$\begin{array}{llll}\text { Rayon moyen : } & \mathrm{R}=43 \cdot 10^{-3} \mathrm{~m} & \text { Module de Young : } \mathrm{E}=2,0.10^{11} \mathrm{~N} \cdot \mathrm{m}^{-2} \\ \text { Longueur: } & \mathrm{L}=10 \mathrm{~m} & \text { Coef. de Poisson : } & \mathrm{v}=0,28 \\ \text { Epaisseur: } & \mathrm{h}=3,2 \cdot 10^{-3} & \text { Densite: } & \rho=7800 \mathrm{Kg} \cdot \mathrm{m}^{-3}\end{array}$

Tableau 1 : Fréquences correspondant au mode transverse $\mathrm{n}=2$

\begin{tabular}{|c|c|c|}
\hline Modes (Long., Trans.) & Cond. Libre-Libre & Cond. Appuyée-Appuyée \\
\hline$(0,2)$ & $1123,93 \mathrm{~Hz}$ & $1123,93 \mathrm{~Hz}$ \\
\hline$(1,2)$ & $1123,94 \mathrm{~Hz}$ & $1123,98 \mathrm{~Hz}$ \\
\hline$(2,2)$ & $1124,04 \mathrm{~Hz}$ & $1124,12 \mathrm{~Hz}$ \\
\hline$(3,2)$ & $1124,24 \mathrm{~Hz}$ & $1124,38 \mathrm{~Hz}$ \\
\hline$(4,2)$ & $1124,56 \mathrm{~Hz}$ & $1124,76 \mathrm{~Hz}$ \\
\hline$(5,2)$ & $1125,01 \mathrm{~Hz}$ & $1125,29 \mathrm{~Hz}$ \\
\hline
\end{tabular}

On s'aperçoit ainsi qu'à partir d'une certaine longueur de conduite, de l'ordre de $10 \mathrm{~m}$, la présence des modes longitudinaux n'affecte plus la frequence des modes transverses. Nous pouvons donc conclure qu'à un mode transverse correspond une infinité de modes longitudinaux dont les fréquences propres sont très voisines de celle du mode transverse.

Si l'on considère maintenant l'équation du $8^{\text {ième }}$ ordre en $\mathrm{k}$, celle-ci peut se résoudre en fixant les valeurs de $n$ et de $\omega$. Le calcul des valeurs de $k$, devrait nous permettre, d'une part d'estimer les vitesses de propagation des différents modes et d'autre part de connaître la valeur qu'il faudra introduire dans le modèle. On aboutit, en résolvant cette équation, à une solution comportant 4 paires de racines $(\mathrm{k},-\mathrm{k})$ qui peuvent être réelles, imaginaires pures ou complexes selon les valeurs de $n$ et de $\omega$.

Le tableau ci-dessous (Tableau 2) présente les 4 solutions obtenues en résolvant l'équation de dispersion en $\mathrm{k}^{8}$ dans le cas de la coque définie précédemment, pour $\mathrm{n}=2$ et $\mathrm{Fn}$ donnée.

Tableau 2: Nombre d'onde théorique pour le mode $\mathrm{n}=2$

\begin{tabular}{|c|c|c|c|c|}
\hline Fréquence Fn & $\mathrm{k}_{1}$ & $\mathrm{k}_{2}$ & $\mathrm{k}_{3}$ & $\mathrm{k}_{4}$ \\
\hline $1123,93 \mathrm{~Hz}$ & 0,00 & $\mathrm{i} .4,20$ & $-100,97+\mathrm{i} .119,82$ & $100,97+\mathrm{i} .119,82$ \\
\hline $1123,98 \mathrm{~Hz}$ & 0,31 & $\mathrm{i} .4,21$ & $-100,97+\mathrm{i} .119,82$ & $100,97+\mathrm{i} .119,82$ \\
\hline $1124,12 \mathrm{~Hz}$ & 0,63 & $\mathrm{i} .4,24$ & $-100,97+\mathrm{i} .119,82$ & $100,97+\mathrm{i} .119,82$ \\
\hline $1124,38 \mathrm{~Hz}$ & 0,94 & $\mathrm{i} .4,30$ & $-100,97+\mathrm{i} .119,82$ & $100,97+\mathrm{i} .119,82$ \\
\hline $1124,76 \mathrm{~Hz}$ & 1,26 & $\mathrm{i} .4,38$ & $-100,97+\mathrm{i} .119,82$ & $100,97+\mathrm{i} .119,82$ \\
\hline $1125,29 \mathrm{~Hz}$ & 1,57 & $\mathrm{i} .4,48$ & $-100,97+\mathrm{i} .119,82$ & $100,97+\mathrm{i} .119,82$ \\
\hline
\end{tabular}

Ces premiers résultats ont été trouvés avec un programme similaire à celui utilisé auparavant . Le nombre d'onde $\mathrm{k}_{1}$ est conforme aux conditions aux limites de type appuyée-appuyée. Par contre, nous n'obtenons pas la solution en "ik $\mathbf{k}_{1}$ définissant en complément de $\mathrm{k}_{\mathbf{l}}$ la déformée d'une poutre en libre-libre $\left(\mathrm{k}_{2} \neq \mathrm{ik}\right)_{1}$. Dans le cas d'un cylindre infini, seule la solution purement propagative est à retenir, elle évolue de façon importante avec l'ordre du mode longitudinal pour une plage de fréquence très restreinte. La célérité des ondes de flexion associées varie dans les même proportions.

\section{3 - EXPERIMENTATIONS :}

\section{1 - Introduction :}

Dans l'étude théorique précédente, nous considérions toujours que, pour un tuyau droit, le champ de déplacement avait une amplitude constante $(\mathrm{U}, \mathrm{V}, \mathrm{W}$ ) le long de l'axe du cylindre (quelle que soit sa longueur). Nous nous proposons de vérifier s'il n'existe pas une perte avec la distance et dans ce cas d'en trouver experimentalement une fonction pour l'approximer, et ce pour un mode $n$ donné.

Ainsi $(\mathrm{U}, \mathrm{V}, \mathrm{W})=$ Constante devient $\left(\mathrm{U}_{\mathrm{n}}, \mathrm{V}_{\mathrm{n}}, \mathrm{W}_{\mathrm{n}}\right)=\mathrm{f}(\mathrm{x})$, pour un tube droit.

D'autre part, nous cherchons à connaître l'influence que peut avoir une singularité de type Coude à $45^{\circ}$, Coude à $90^{\circ}$, Cintrage, Té... sur le signal qui se propage et plus particulièrement sur son amplitude.

Là encore, nous nous proposons de quantifier cette atténuation par des mesures expérimentales. 


\section{2 - Principe et résultats des mesures :}

Des essais réalisés précédemment sur des tubes similaires à celui dont les caractéristiques sont définies en 1.3 , mais de longueur $\mathrm{L}=47 \mathrm{~m}$ et $\mathrm{L}=63 \mathrm{~m}$, ont permis de montrer que le mode de propagation transverse $n=2$ (situé autour de $1168 \mathrm{~Hz}$ ) était prépondérant (Figure 1). On s'attachera donc à définir une loi de décroissance de l'amplitude de ce mode avec la distance, en essayant de ne prendre en compte lors de la mesure, que l'onde incidente.

Pour cela, on positionne plusieurs capteurs accélérométriques (suivant les 3 directions) en différents points répartis le long de l'axe du tube. Nous mesurons simultanément en chaque point, sur la courbe de réponse en fréquence, l'amplitude correspondante au pic du mode $\mathbf{n}=2$. En regroupant l'ensemble des points de mesures issus de plusieurs essais, on obtient, suivant chaque direction, une décroissance de l'amplitude de ce mode avec la distance. On peut établir une loi mathématique pour cette décroissance en utilisant une méthode des moindre carrés par rapport aux points expérimentaux.

Les premiers résultats concernant le tube droit sont présentés ci-dessous (Figure 2). On constate dans ce cas de mesure suivant la direction radiale, que l'atténuation est relativement importante dans les premiers mètres après l'excitation. Ensuite, elle décroît beaucoup plus lentement. Ceci pourrait être dû à des phénomènes non linéaires et transitoires causés par différents trains d'ondes de célérité différente. Des mesures complémentaires, ainsi que celles concernant les singularités sont en cours.

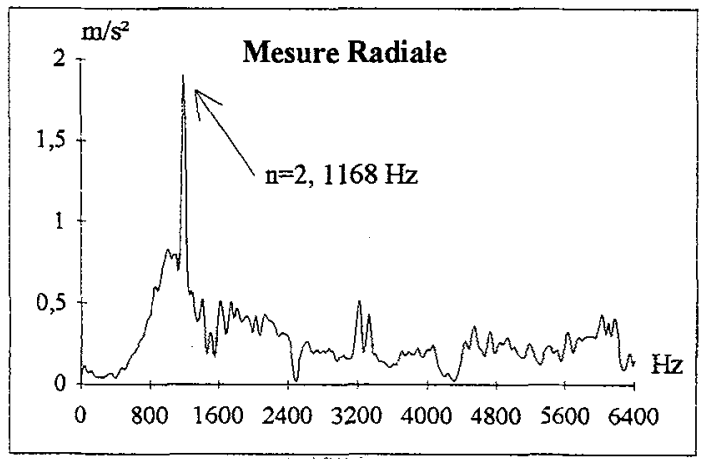

Figure 1: Autospectre d'Accélération Tube droit $47 \mathrm{~m}$ - Mesure radiale

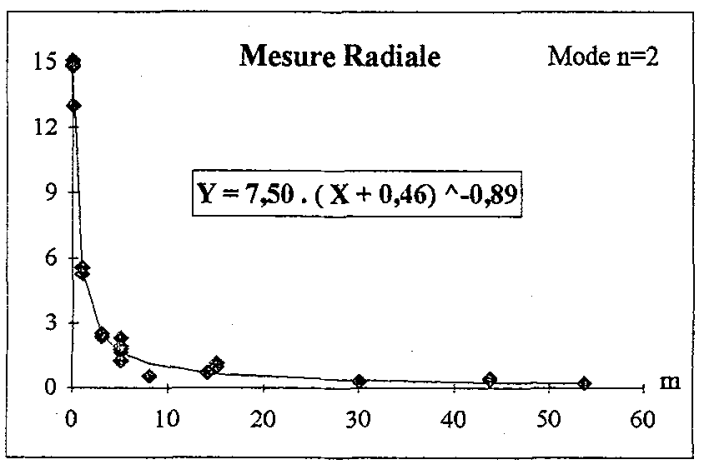

Figure 2: Approximation de la loi de décroissance de l'amplitude avec la distance Tube droit $63 \mathrm{~m}$ - Mesure radiale - Mode $\mathrm{n}=2$

\section{4 - CONCLUSION :}

Le programme de calcul nous a permis d'appréhender les modes de propagation à considérer. Les essais ont confirmé la prépondérance de certains modes ainsi que le bon accord entre les fréquences théoriques et expérimentales. Une loi de décroissance de l'amplitude avec la distance a pu être dégagée.

Cette loi de décroissance ainsi que les atténuations propres à chaque singularité pourront être intégrées dans un programme de simulation numérique. Celui-ci nous permettra alors de connaître l'amplitude, en un point donné, d'un signal issu d'une excitation donnée après parcours d'une portion de conduite en présence ou non de singularités.

\section{Références :}

[1] : S. Timoshenko \& S. Woinowsky-Krieger, "Theorie des plaques et coques", $2^{\text {nde }}$ édition, (1961)

[2] : F.Fahy, "Sound and structural vibration", Academic Press, (1985)

[3] : A. W. Leissa, "Vibration of shells", NASA SP-288, (1973)

[4] : C. Y. Glandier, "Wave-vector analysis of the vibration of thin cylindrical shells", Thesis of Georgia Institute of Technology, USA, (1991) 